\title{
CARACTERIZAÇÃO ENERGÉTICA DA MADEIRA DE TRÊS MATERIAIS GENÉTICOS DE Eucalyptus sp.
}

\author{
Eleandro José Brun ${ }^{1 *}$, Aline Patricia Bersch ${ }^{2}$, Flávia Alves Pereira ${ }^{3}$, Dimas Agostinho Silva ${ }^{4}$, Yan Rafael de \\ $\mathrm{Barba}^{5}$, Jeferson Ricardo Dorini Junior ${ }^{5}$ \\ ${ }^{1}$ Universidade Tecnológica Federal do Paraná, Curso de Engenharia Florestal e Programa de Pós-graduação em Agroecossistemas, Dois \\ Vizinhos, Paraná, Brasil - eleandrobrun.utfpr@gmail.com. \\ ${ }^{2}$ Universidade Paranaense, Especialização em Perícia e Auditoria Ambiental, Capanema, Paraná, Brasil - aline_bersch@ hotmail.com. \\ ${ }^{3}$ Universidade Tecnológica Federal do Paraná, Curso de Engenharia Florestal, Dois Vizinhos, Paraná, Brasil - flaviapereira@utfpr.edu.br. \\ ${ }^{4}$ Universidade Federal do Paraná, Departamento de Ciências Florestais, Curitiba, Paraná, Brasil - dimas.agostinho.silva@ gmail.com. \\ ${ }^{5}$ Universidade Tecnológica Federal do Paraná, Dois Vizinhos, Paraná, Brasil - yanrafaeldebarba@ hotmail.com, \\ jefersondorinijunior@gmail.com.
}

Recebido para publicação: 10/04/2017 - Aceito para publicação: 24/11/2017

\begin{abstract}
Resumo
Entre as características da madeira mais indicadas para geração de energia, estão: a massa específica, o poder calorífico e o teor de carbono fixo. O objetivo deste trabalho foi realizar a caracterização energética da madeira de três materiais genéticos de Eucalyptus sp.: clones H-13 e GFMO-27 e o híbrido E. pellita x E. tereticornis, plantados em área experimental em Dois Vizinhos, sudoeste do Paraná. Esses materiais foram escolhidos por se destacarem em incremento médio anual em volume aos cinco anos de idade entre quatorze materiais genéticos plantados em uma mesma área. Três árvores de cada um dos materiais genéticos foram abatidas e três discos de madeira de cada árvore foram retirados a 0\%, 50\% e 100\% da altura comercial. Esses discos foram descascados e pesados. Posteriormente, determinou-se a massa específica básica (NBR 11941:2003), o poder calorífico (NBR 8633:1984), a análise química imediata (MB 15) e a energia disponível na biomassa. Os dados foram analisados através de análise de variância e teste de Tukey. Os valores de massa específica básica e teor de cinzas não apresentaram diferença estatística entre os materiais. O valor de massa específica ficou entre 0,49 e $0,55 \mathrm{~g} / \mathrm{cm}^{3}$ e o teor de cinzas entre 0,57 e $0,60 \%$. O clone $\mathrm{H}-13$ apresentou o maior valor de materiais voláteis entre os três materiais genéticos e, consequentemente, o menor valor de carbono fixo. O clone GFMO-27 apresentou maior valor médio de poder calorífico. Contudo, os valores de estimativa de energia disponível na madeira não apresentaram diferença estatística entre os materiais genéticos.
\end{abstract}

Palavras-chave: Lenha, poder calorífico, massa específica, biomassa florestal.

\begin{abstract}
Characterization energy of wood of three genetic materials of Eucalyptus sp. Among the wood's most suitable characteristics for power generation, there is density, calorific value and content of fixed carbon. The aim of this work was to execute the energy characterization of three genetic material of Eucalyptus sp.: clones H-13 and GFMO-27, and the hybrid E. pellita x E. tereticornis, planted in the municipality of Dois Vizinhos, southwest of Paraná, Brazil. These three materials were chosen by outstanding among the others in average annual increment in volume at the age of five. They were chosen among fourteen genetic materials planted in the same area. Three trees of each genetic material were shot down, and three wooden discs of every tree were selected at $0 \%, 50 \%$ and $100 \%$ of commercial height. These discs were peeled and weighted. Subsequently, the basic density (NBR 11941:2003), the calorific value (NBR 8633:1984), the immediate chemical analysis (MB 15 ) and the available energy on wood were determined. Data were analyzed by using the analysis of variance and the Tukey's test. The values of basic density and ash content showed no statistical difference among the materials. The clone H-13 presented the largest value of volatile materials among the three genetic materials and, consequently, lower fixed carbon. The clone GFMO-27 showed the highest average value of calorific value. However, the estimated values of energy available in the wood showed no statistical difference among the genetic materials.

Keywords: Firewood, calorific value, specific mass, forest biomass.
\end{abstract}

\section{INTRODUÇÃO}

O aumento do consumo mundial de energia baseada em combustíveis fósseis direcionou a humanidade a uma matriz energética insegura, cara e prejudicial ao meio ambiente. Esse fato tem levado vários países a intensificarem o aproveitamento de outras fontes energéticas, principalmente as renováveis, como a madeira (BRITO, 2007). A madeira é o combustível mais antigo empregado para gerar energia e, entre suas vantagens, está o baixo custo e os menores impactos ambientais, quando comparados aos combustíveis não-renováveis (NASCIMENTO; BIAGGIONI, 2010).

FLORESTA, Curitiba, PR, v. 48, n. 1, p. 87-92, jan./marc. 2018.

Brun. E. J, et.al

ISSN eletrônico 1982-4688

DOI: $10.5380 /$ rf.v48 i1.51673 
Segundo a EMBRAPA (2008), novas técnicas de plantio e manejo de espécies florestais foram desenvolvidas visando atender a grande demanda por madeira nos segmentos de painéis reconstituídos, móveis, construção civil, indústria de papel, lenha e carvão vegetal.

A expressão "florestas energéticas" foi criada para definir os maciços florestais que têm como finalidade produzir maior quantidade de energia por área em menor espaço de tempo. Rotações de 2 a 4 anos com menor espaçamento, geralmente $2 \times 2 \mathrm{~m}$, resultam em maior número de árvores por hectare. Normalmente, as espécies mais adaptadas para tal finalidade são as do gênero Eucalyptus (NOGUEIRA; LORA, 2003). Este engloba mais de 600 espécies e outras centenas de materiais genéticos melhorados (clones e híbridos), adaptados às mais diferentes condições de clima e solo, oferecendo uma ampla gama de usos.

O uso da madeira para geração de energia não é restritivo, ou seja, não há espécies que não possam ser utilizadas. Entretanto, algumas características intrínsecas, como a massa específica, podem indicar espécies mais adequadas para esta finalidade. A massa específica é um dos índices de qualidade mais representativos, estando diretamente ligado à produção de matéria seca.

A análise do poder calorífico, quantidade de voláteis, carbono fixo e teor de cinzas também são parâmetros de grande importância para uso energético. O poder calorífico expressa a quantidade de calor liberado na queima completa de uma unidade de massa do combustível e é influenciado por teores de extrativos e lignina (SANTOS et al., 2011). A maior quantidade de carbono fixo na madeira acarreta em uma queima mais lenta, enquanto maiores teores de matérias voláteis propiciam uma degradação mais acelerada. As cinzas são formadas por materiais inorgânicos e não contribuem para o aumento do poder calorífico.

Neste contexto, a implantação de testes e seleção de materiais genéticos com maior incremento e melhores propriedades energéticas são relevantes para atender a demanda de geração de energia. O objetivo deste trabalho foi avaliar as propriedades energéticas da madeira dos clones H-13 e GFMO-27 e do híbrido E. pellita x E. tereticornis.

\section{MATERIAL E MÉTODOS}

O material foi coletado na Estação Experimental TUME (Teste de Uso Múltiplo de Eucalipto), pertencente à Universidade Tecnológica Federal do Paraná, Campus Dois Vizinhos, com coordenadas centrais de 534'30"' W e 25॰44'35', S e altitude média de 509 metros.

O clima característico da região, segundo a classificação de Köppen, é o Cfa, subtropical, com temperatura variando entre $18{ }^{\circ} \mathrm{C} \mathrm{a}-3{ }^{\circ} \mathrm{C}$ no mês mais frio. A ocorrência de geadas é frequente e a temperatura do mês mais quente ultrapassa $23{ }^{\circ} \mathrm{C}$. As chuvas são bem distribuídas em todos os meses do ano, sem ocorrência de estação seca, com pluviosidade anual média de 2025 mm (INSTITUTO AGRONÔMICO DO PARANÁ, 2015). O solo é classificado como Latossolo Vermelho Distroférrico típico (EMPRESA BRASILEIRA DE PESQUISA AGROPECUÁRIA, 2006).

Os materiais genéticos de Eucalyptus sp. do estudo foram plantados em área anteriormente agrícola, em espaçamento 3 × 2 m, que compunha parcelas experimentais contendo 180 plantas (10 linhas de 18 plantas), e em solo preparado via escarificação mecânica na linha de plantio e adubação de plantio igual a 300 g de NPK 6-30-6, aplicada via coveta lateral logo após o plantio. Os tratos culturais (controle de formigas e plantas competidoras) foram realizados rotineiramente durante o primeiro e segundo ano após o plantio.

A escolha dos materiais genéticos para este estudo ocorreu a partir de um inventário florestal realizado na estação experimental em julho de 2014, onde os 14 materiais genéticos foram avaliados quando o plantio tinha 4,5 anos de idade. Três materiais genéticos se destacaram com maior incremento médio anual (IMA) em volume: o clone H-13, o híbrido E. pellita x E. tereticornis e o clone GFMO-27, com IMA de 68, 62 e 60 mªha/ano, respectivamente. Os dados foram obtidos mediante inventário por censo e cubagem, pelo método de Smalian, de cinco árvores por parcela, uma em cada classe de diâmetro.

Das cinco árvores abatidas para cubagem, foram escolhidas três de cada material genético entre as mais centrais de cada parcela de plantio. O diâmetro do tronco foi determinado em diferentes posições: $0,1 \mathrm{~m}, 0,3 \mathrm{~m}$ e, então, de metro a metro até a altura comercial (diâmetro mínimo de cinco centímetros).

Foram retirados três discos de madeira com 5 centímetros de espessura de cada árvore nas alturas relativas de $0 \%$ (base), $50 \%$ (meio) e 100\% (topo) da altura comercial do tronco. Esses discos, ainda no campo, foram descascados e pesados em uma balança digital para a obtenção da massa úmida e, em seguida, levados para uma estufa de ventilação forçada à temperatura de $103^{\circ} \mathrm{C} \pm 2^{\circ} \mathrm{C}$ até obtenção de massa constante.

A massa específica básica da madeira foi determinada seguindo a norma técnica NBR 11941 (ASSOCIAÇÃO BRASILEIRA DE NORMAS TÉCNICAS, 2003), na qual os discos de madeira secos em estufa foram pesados e a massa obtida foi dividida pelo volume verde obtido em campo no momento do corte das árvores.

A determinação do poder calorífico superior (PCS), em MJ kg-1, foi realizada no Laboratório de Energia da Biomassa da Universidade Federal do Paraná, Campus Curitiba, com utilização de uma bomba calorimétrica adiabática, seguindo a NBR 8633 (ASSOCIAÇÃO BRASILEIRA DE NORMAS TÉCNICAS, 1984). Para 
calcular o poder calorífico inferior (PCI), foi utilizada a equação 1, com valor de hidrogênio de 6\%. Para calcular o poder calorífico útil, foi utilizada a equação 2, com teor de umidade de $20 \%$ na base úmida (valor médio no qual a madeira é utilizada para lenha na queima em aviários).

$$
P C I=P C S-\left(600 * 9-\left(\frac{H}{100}\right)\right)
$$

em que: $\mathrm{PCI}=$ poder calorífico inferior da madeira $\left(\mathrm{MJ} \mathrm{kg}^{-1}\right) ; \mathrm{PCS}=$ poder calorífico superior da madeira $\left(\mathrm{MJ} \mathrm{kg}^{-1}\right)$; $\mathrm{H}=$ hidrogênio $(\%)$.

$$
P C U=P C I *(100-U / 100)-6 * U
$$

em que: $\mathrm{PCU}=$ poder calorífico útil da madeira $\left(\mathrm{MJ} \mathrm{kg}^{-1}\right)$; $\mathrm{PCI}=$ poder calorífico inferior da madeira $\left(\mathrm{MJ} \mathrm{kg}^{-1}\right)$; $\mathrm{U}=$ umidade $(\%)$, na base úmida.

A análise química imediata (teores de materiais voláteis, carbono fixo e cinzas) foi realizada no Laboratório de Tecnologia da Madeira na Universidade Tecnológica Federal do Paraná, Campus Dois Vizinhos, e seguiu o Método Brasileiro - MB 15.

$\mathrm{O}$ incremento em massa seca, que corresponde à biomassa de madeira por área, foi estimado através da equação 3 .

$$
I M S=I M A * r b
$$

em que: IMS = incremento em massa seca $\left(\mathrm{t} \mathrm{ha}^{-1} \mathrm{ano}^{-1}\right) ; \mathrm{IMA}=$ incremento médio anual $\left(\mathrm{m}^{3} \mathrm{ha}^{-1} \mathrm{ano}^{-1}\right) ; \mathrm{rb}=$ massa específica básica $\left(\mathrm{kg} \mathrm{m}^{-3}\right)$.

Para a estimativa da quantidade de energia disponível por hectare ano ${ }^{-1}$, expressa em MJ, foi utilizada a equação 4 .

$$
E D=I M S * P C U
$$

em que: $\mathrm{ED}=$ energia disponível $\left(\mathrm{MJ} \mathrm{ha}^{-1} \mathrm{ano}^{-1}\right) ; \mathrm{IMS}=$ incremento em massa seca $\left(\mathrm{t} \mathrm{ha}^{-1}\right.$ ano $\left.^{-1}\right) ; \mathrm{PCU}=$ poder calorífico útil da madeira $\left(\mathrm{MJ} \mathrm{ton}^{-1}\right)$.

O delineamento inteiramente casualizado, disposto em esquema fatorial, foi utilizado para a análise da madeira. Foram três árvores de cada material genético, três materiais (clone H-13, clone GFMO-27 e E. pellita x E. tereticornis) e três discos por árvore (0, 50 e $100 \%$ da altura comercial), totalizando 27 amostras.

Os resultados foram submetidos à análise de variância (ANOVA) para verificar se houve diferença entre as alturas e os materiais genéticos avaliados. Quando estabelecidas diferenças significativas, foi realizado o teste de Tukey a 5\% de probabilidade de erro e 95\% de significância para comparar os tratamentos entre si. As análises dos dados foram processadas no software estatístico ASSISTAT® versão 7.7, sendo considerado como tratamento as alturas do tronco e os materiais genéticos.

A análise de correlação entre as variáveis massa específica básica, poder calorífico útil e análise química imediata foi feita pelo coeficiente de correlação linear de Pearson, utilizando o software Microsoft Office Excel.

\section{RESULTADOS}

A análise de variância do delineamento experimental utilizado mostrou que somente o efeito de material genético foi significativo, em nível de $5 \%$ de probabilidade de erro, para o poder calorífico útil, teor de materiais voláteis e de carbono fixo. Os valores médios obtidos para as propriedades da madeira dos materiais genéticos clone GFMO-27, híbrido E. pellita x E. tereticornis e clone H-13, além do teste de comparação múltipla realizado, estão apresentados na Tabela 1.

Tabela 1. Valores médios da massa específica básica, poder calorífico útil, análise imediata (materiais voláteis, cinzas e carbono fixo), produtividade de biomassa e de energia dos clones GFMO-27 e clone H-13 e híbrido E. pellita x E. tereticornis.

Table 1. Mean values of basic specific mass, useful calorific value, immediate analysis (volatile materials, ash and fixed carbon), biomass and energy yield of clone GFMO-27 and clone $\mathrm{H}-13$ and hybrid E. pellita $\mathrm{x}$ E. tereticornis.

\begin{tabular}{llll}
\hline Propriedade / Material & GFMO-27 & E. pellita $\boldsymbol{x}$ E. tereticornis & H-13 \\
\hline Massa específica básica $\left(\mathrm{g} \mathrm{cm}^{-3}\right)$ & $0,50 \mathrm{a}$ & $0,55 \mathrm{a}$ & $0,49 \mathrm{a}$ \\
Poder calorífico útil $\left(\mathrm{MJ} \mathrm{kg}^{-1}\right)$ & $14,25 \mathrm{a}$ & $14,04 \mathrm{ab}$ & $13,97 \mathrm{~b}$ \\
\hline
\end{tabular}

FLORESTA, Curitiba, PR, v. 48, n. 1, p. 87-92, jan./marc. 2018.

Brun. E. J, et.al

ISSN eletrônico 1982-4688

DOI: $10.5380 /$ rf.v48 i1.51673 


\begin{tabular}{llll}
\hline Materiais voláteis (\%) & $83,17 \mathrm{~b}$ & $83,81 \mathrm{~b}$ & $86,16 \mathrm{a}$ \\
Cinzas (\%) & $0,60 \mathrm{a}$ & $0,58 \mathrm{a}$ & $0,57 \mathrm{a}$ \\
Carbono fixo (\%) & $16,23 \mathrm{a}$ & $15,61 \mathrm{a}$ & $13,27 \mathrm{~b}$ \\
Produtividade da biomassa (ton ha-1 $\left.\mathrm{ano}^{-1}\right)$ & $30,33 \mathrm{a}$ & $34,24 \mathrm{a}$ & $33,22 \mathrm{a}$ \\
Est. de energia disponível $\left(\mathrm{MJ} \mathrm{ha}^{-1} \mathrm{ano}^{-1}\right)$ & $432.832 \mathrm{a}$ & $481.435 \mathrm{a}$ & $464.425 \mathrm{a}$ \\
\hline
\end{tabular}

As médias seguidas pela mesma letra na linha não diferem estatisticamente entre si pelo teste de Tukey a 5\% de probabilidade de erro.

A análise da correlação entre as variáveis pelo coeficiente de correlação linear de Pearson apresentou os resultados demonstrados na Tabela 2.

Tabela 2Resultados da correlação linear de Pearson entre as propriedades analisadas.

Table 2. Results of Pearson linear correlation among the properties analyzed.

\begin{tabular}{|c|c|c|c|c|c|c|}
\hline Propriedade & $\begin{array}{l}\text { Massa } \\
\text { básica }\end{array}$ & esp. & $\overline{P C U}$ & Mat. voláteis & Cinzas & Carbono fixo \\
\hline $\begin{array}{l}\text { Massa esp. } \\
\text { básica }\end{array}$ & 1 & & $0,05^{\mathrm{ns}}$ & $-0,011^{\mathrm{ns}}$ & $0,107^{\mathrm{ns}}$ & $0,007^{\mathrm{ns}}$ \\
\hline PCU & - & & 1 & $-0,371 *$ & 0,198 & $0,367^{*}$ \\
\hline Mat. voláteis & - & & - & 1 & $-0,262 *$ & -1 \\
\hline Cinzas & - & & - & - & 1 & 0,232 \\
\hline Carbono fixo & - & & - & - & - & 1 \\
\hline
\end{tabular}

* significativo ao nível de 5\% de probabilidade de erro; ns: não significativo.

\section{DISCUSSÃO}

A massa específica básica entre os três materiais genéticos variou de 0,49 a $0,55 \mathrm{~g} / \mathrm{cm}^{3}$, não apresentando diferenças significativas. Resultados semelhantes foram obtidos por Alzate et al. (2005) ao estudar clones de E. grandis, E. saligna e de E. grandis $x$ E. urophylla. Esses autores verificaram que a massa específica básica média dessas madeiras foi de 0,46, 0,47 e 0,49 $\mathrm{g} / \mathrm{cm}^{3}$, respectivamente. Lima et al. (2011) observaram o valor de $0,475 \mathrm{~g} / \mathrm{cm}^{3}$ para $E$. benthamii com seis anos de idade, semelhante ao encontrado neste trabalho.

Os valores médios de poder calorífico útil variaram de $13,97 \mathrm{MJ} \mathrm{kg}^{-1}$ no clone $\mathrm{H}-13$ a $14,25 \mathrm{MJ} \mathrm{kg}^{-1}$ no clone GFMO-27. Esses resultados podem ser explicados pelos valores encontrados na análise química imediata, na qual o clone GFMO-27 apresentou valores maiores de carbono fixo do que o clone H-13. Isso mostra a correlação positiva entre essas duas propriedades. Quanto maior o teor de carbono fixo, maior o poder calorífico, uma vez que a madeira libera energia por mais tempo durante o processo de combustão. Soares et al. (2014), ao estudar a correlação entre as propriedades da madeira e do carvão vegetal de híbridos de eucalipto, também encontraram correlação positiva entre o teor de carbono fixo e o poder calorífico.

Quanto ao teor de materiais voláteis, o clone GFMO-27 e o híbrido E. pellita x E. tereticornis não diferiram significativamente entre si, possuindo médias de materiais voláteis de 83,17 e 83,81\%, respectivamente. Enquanto isso, o clone H-13 diferiu significativamente, apresentando média de $86,16 \%$. Esses resultados podem ser explicados se comparados com os valores de carbono fixo obtidos neste trabalho, em que os valores médios, para o clone GFMO-27 e para o híbrido E. pellita x E. tereticornis, foram maiores estatisticamente em relação aos valores de carbono fixo do clone H-13. Os valores de materiais voláteis e carbono fixo são inversamente proporcionais. Resultados semelhantes foram observados por Chaves et al. (2013), que obtiveram valores de materiais voláteis entre 83 e $85 \%$ ao avaliar três clones de Eucalyptus com sete anos. Vale et al. (2002), estudando 47 espécies madeireiras do Cerrado, verificaram médias de materiais voláteis entre 74,62 a 81,20\%. Esses valores foram menores do que os obtidos no presente estudo. Provavelmente, essa variação se deve pela diferença entre as espécies e materiais genéticos pesquisados.

O teor de cinza não diferiu significativamente entre os três materiais genéticos, variando entre 0,57 e 0,60\%. Chaves et al. (2013), ao testar três clones de Eucalyptus, verificaram valores de cinza mais baixos, entre 0,29 e $0,45 \%$. Neves et al. (2013) também observaram valores entre 0,22 e 0,42\% para clones de Eucalyptus.

Os teores de carbono fixo do clone GFMO-27 e do híbrido E. pellita x E. tereticornis não diferiram significativamente, apresentando valores de 16,23 e $15,61 \%$, respectivamente. O clone $\mathrm{H}-13$ diferiu estatisticamente dos demais, obtendo média de $13,27 \%$. Essa diferença pode ser explicada pelo teor de materiais voláteis, no qual o clone H-13 também diferiu significativamente dos demais materiais genéticos. O H-13 apresentou valores mais altos para este aspecto e, consequentemente, valores mais baixos de carbono fixo, pois essas duas variáveis são inversamente proporcionais. Resultados semelhantes foram constatados por Silva et al. (2015) que, avaliando o E. benthamii com cinco anos, chegaram ao valor médio de carbono fixo de 
15,78\%. Chaves et al. (2013) também obtiveram valores semelhantes aos encontrados nesse trabalho em teste de três clones de Eucalyptus, no qual os valores de carbono fixo variaram entre 15,72 e 16,08\%.

Os valores médios para massa seca da madeira não diferiram significativamente entre os materiais genéticos. Os valores variaram de 30,33 ton ha ${ }^{-1}$ ano $^{-1}$ no clone GFMO-27 a 34,24 ton.ha ${ }^{-1}$ ano $^{-1}$ no híbrido E. pellita x E. tereticornis. Santos et al. (2012), ao testar três clones de E. urophylla, observaram que um se destacou com maior massa de matéria seca. Os pesquisadores explicam que isso ocorre devido ao fato de esse clone ter um alto IMA e densidade básica superior aos outros dois clones.

Os valores médios obtidos nos cálculos da estimativa de energia disponível não apresentaram diferença significativa entre os três materiais genéticos. Os valores variaram de $432.832 \mathrm{MJ} \mathrm{ha}^{-1}$ ano $^{-1}$ no clone GFMO-27 a 481.435 MJ ha ${ }^{-1} \mathrm{ano}^{-1}$ no híbrido E. pellita x E. tereticornis. Apesar de o valor médio de PCU do clone GFMO-27 ter sido maior, seu resultado de energia disponível não diferiu significativamente dos demais materiais genéticos; fato explicado pelo valor de massa seca de madeira por hectare ter sido menor do que os demais materiais. Santos et al. (2012) verificaram que um dos clones de E. urophylla apresentou maior quantidade de energia disponível, fato relacionado, pelos autores, à elevada massa de matéria seca desse clone em relação aos outros dois. Lima et al. (2011) observaram valores mais elevados de produtividade energética anual para o E. benthamii, sendo $323 \mathrm{Gcal} \mathrm{ha}^{-1} \mathrm{ano}^{-1}$, ou seja, 1.351.429 $\mathrm{MJ} \mathrm{ha}^{-1} \mathrm{ano}^{-1}$.

Analisando os resultados de correlação entre as propriedades da madeira avaliadas, pode-se explicar a correlação positiva entre o poder calorífico útil e o carbono fixo pelo fato de que quanto mais carbono há na madeira (principal fonte de energia para a combustão), maior é o poder calorífico. Consequentemente, a correlação do poder calorífico útil com os materiais voláteis é negativa. A correlação negativa entre os materiais voláteis e as cinzas ocorre, pois o material que é volatizado não resulta em resíduos sólidos após a combustão (ou seja, as cinzas). Portanto, quanto maior o teor de materiais voláteis na madeira, menor o teor de cinzas.

Resultados semelhantes aos deste trabalho foram encontrados por Soares et al. (2014). Ao estudar o híbrido Eucalyptus grandis $x$ Eucalyptus urophylla, os pesquisadores verificaram que o teor de carbono fixo e o poder calorífico correlacionaram-se negativamente com o teor de materiais voláteis. Silva et al. (2015) também verificou correlação negativa entre o teor de materiais voláteis e o teor de carbono fixo e cinzas.

\section{CONCLUSÕES}

Pelos resultados obtidos, conclui-se que:

- A massa específica básica, o teor de cinzas, o incremento em massa seca/produtividade de biomassa e a energia disponível não diferiram entre os materiais genéticos estudados.

- Os valores de poder calorífico útil foram maiores no clone GFMO-27 e menores no clone H-13.

- Os teores de materiais voláteis foram maiores no clone H-13.

- Os valores de carbono fixo foram menores no clone H-13.

- Ambos os materiais genéticos pesquisados têm potencial para geração de energia pelo uso da sua biomassa.

\section{REFERÊNCIAS}

ALZATE, S. B. A.; TOMAZELLO FILHO, M.; PIEDADE, S. M. de S. Variação longitudinal da densidade básica da madeira de clones de Eucalyptus grandis Hill ex Maiden, E. saligna Sm. e E. grandis x urophylla. Scientia Forestalis, n. 68, p. 87-95, 2005.

ASSOCIAÇÃO BRASILEIRA DE NORMAS TÉCNICAS - ABNT. NBR 8633 - Carvão vegetal Determinação do poder calorífico. Rio de Janeiro, 1984, 13p.

ASSOCIAÇÃO BRASILEIRA DE NORMAS TÉCNICAS - ABNT. NBR 11941 - Determinação da densidade básica em madeira. Rio de Janeiro, 2003, 6p.

CHAVES, A. M. B.; VALE, A. T. do; MELIDO, R. C. N.; ZOCH, V. P. Características energéticas da madeira e do carvão vegetal de clones de Eucalyptus spp. Enciclopédia Biosfera, Centro Científico Conhecer - Goiânia, v. 9, n. 17, p. 533-542, 2013.

EMPRESA BRASILEIRA DE PESQUISA AGROPECUÁRIA - EMBRAPA. Sistema Brasileiro de Classificação de Solos. Rio de Janeiro: EMBRAPA-SPI, 2 ed. 2006, 306 p.

EMPRESA BRASILEIRA DE PESQUISA AGROPECUÁRIA - EMBRAPA. Florestas energéticas na matriz de agroenergia brasileira. Brasília: Embrapa, 2008, 14 p.

FLORESTA, Curitiba, PR, v. 48, n. 1, p. 87-92, jan./marc. 2018.

Brun. E. J, et.al

ISSN eletrônico 1982-4688

DOI: $10.5380 /$ rf.v48 i1.51673 
INSTITUTO AGRONÔMICO DO PARANÁ (IAPAR). Classificação Climática. Disponível em: < http://www.iapar.br/modules/conteudo/conteudo.php?conteudo=863>. Acesso em: 16/10/2015.

LIMA, E. A. de; SILVA, H. D. da; LAVORANTI, O. J. Caracterização dendroenergética de árvores de Eucalyptus benthamii. Pesquisa Florestal Brasileira, Colombo, v. 31, n. 65, p. 09-17, 2011.

NASCIMENTO, M. D. do; BIAGGIONI, M. A. M. Avaliação energética do uso de lenha e cavaco de madeira para produção de energia em agroindústria seropédica. Energia na Agricultura, Botucatu, v. 25, n. 3, p. 104 $117,2010$.

NEVES, T. A.; PROTÁSIO, T. de P.; TRUGILHO, P. F.; VALLE, M. L. A.; SOUSA, L. C. de; VIEIRA, C. M. M. Qualidade da madeira de clones de Eucalyptus em diferentes idades para a produção de bioenergia. Revista Ciências Agrárias, v. 56, n. 2, p. 139-148, 2013.

NOGUEIRA, L. A. H.; LORA, E. E. S. Dendroenergia: Fundamentos e Aplicações. Rio de Janeiro: Interciência, 2 ed. 2003, 193p.

SANTOS, L. C.; CARVALHO, A. M. M. L.; PEREIRA, B. L. C.; OLIVEIRA, A. C.; CARNEIRO, A. de C. O.; TRUGILHO, P. F. Propriedades da madeira e estimativas de massa, carbono e energia de clones de Eucalyptus plantados em diferentes locais. Revista Árvore, Viçosa, v. 36, n. 5, p. 971-980, 2012.

SILVA, D. A.; MULLER, B. V.; KUIASKI, E. C.; ELOY, E.; BEHLING, A.; COLAÇO, C. M. Propriedades da madeira de Eucalyptus benthamii para produção de energia. Pesquisa Florestal Brasileira, Colombo, v. 35, n. 84, p. 481-485, 2015.

SOARES, V. C.; BIANCHI, M. L.; TRUGILHO, P. F.; PEREIRA, A. J.; HOFLER, J. Correlações entre as propriedades da madeira e do carvão vegetal de híbridos de eucalipto. Revista Árvore, Viçosa, v. 38, n. 3, p. 543 $-549,2014$.

VALE, A. T. do; BRASIL, M. A. M.; LEÃO, A. L. Quantificação e caracterização energética da madeira e casca de espécies do cerrado. Ciência Florestal, Santa Maria, v. 12, n. 1, p. 71-80, 2002. 